\title{
Predição do comportamento da massa específica das polpas de abacaxi e acerola sob diferentes temperaturas ${ }^{1}$
}

\author{
Maria das Neves Mendonça Alves², Djancley Araújo dos Santos ${ }^{3}$, João Carlos Soares de \\ Melo $^{4}$, Carlos Helaidio Chaves da Costa ${ }^{5}$, Adair Divino Silva Badaró ${ }^{6}$, Regilane Marques \\ Feitosa $^{7}$
}

\begin{abstract}
Resumo: A dependência da massa específica com a temperatura da polpa de fruta é muito importante para as indústrias de alimentos em geral, uma vez que esse dado é essencial na concepção e optimização de várias operações, como no projeto de bombas, trocadores de calor, evaporadores e misturadores. Dessa forma, o objetivo desse trabalho foi determinar experimentalmente a massa específica das polpas de abacaxi e acerola em diferentes temperaturas e estabelecer uma correlação entre essa propriedade com a variação da temperatura de $10,20,30,40$ e $50^{\circ} \mathrm{C}$, utilizando modelos matemáticos para predição desta propriedade. Para a obtenção dos valores da energia de ativação para a massa específica $\left(E_{a}\right)$ foi empregado o método proposto por Tsen e King (2002). Os valores da massa específica diminuíram com $\mathrm{o}$ aumento da temperatura. $\mathrm{O}$ modelo polinomial com três termos apresentou os maiores coeficientes de determinação $\left(R^{2}>0,99\right)$, podendo ser usado para estimar a massa específica das polpas estudadas. A polpa de abacaxi apresentou maior sensibilidade à aplicação das diferentes temperaturas.
\end{abstract}

Palavras chaves: Densidade; Polpa de fruta; Energia de ativação.

\section{Prediction of specific mass behavior in the pineapple and acerola pulps under different temperatures}

\begin{abstract}
The dependence of the specific mass with the temperature of the fruit pulp is very important for the food industries in general, since this is essential in the design and optimization of various operations, such as in the design of pumps, heat exchangers, coolers and mixers. Thus, the objective of this work is to determine experimentally the specific mass of the pineapple and acerola pulps at different temperatures and co-relate this property with the temperature variation of $10,20,30,40$ and $50^{\circ} \mathrm{C}$, using mathematical models to predict this property. To obtain the values of the activation energy for the specific mass $\left(E_{a}\right)$ was employed a proposed method by Tsen and King (2002). The specific mass values decreased with the temperature increase. The polynomial model with three terms presented the highest coefficients of determination $\left(\mathrm{R}^{2}>0,99\right)$, and it can be used to estimate the specific mass studied pulps. The pineapple pulp showed greater sensitivity to different temperatures.
\end{abstract}

Keywords: Density; Pulp fruits; Activation energy.

\footnotetext{
${ }^{1}$ Submetido 01/08/2017 e aprovado em 04/04/2018

${ }^{2}$ Técnico em Eletrotécnica; Estudante, Instituto Federal de Educação, Ciência e Tecnologia do Rio Grande do Norte (IFRN), Caicó - Rio Grande do Norte, CEP: 59300-000; E-mail: nevinha.alves2014@gmail.com

${ }^{3}$ Técnico em Eletrotécnica; Estudante, Instituto Federal de Educação, Ciência e Tecnologia do Rio Grande do Norte (IFRN), Caicó - Rio Grande do Norte, CEP: 59300-000; E-mail: djancleysentinela@ outlook.com

${ }^{4}$ Doutor em Engenharia de Processos; Técnico em Química, Instituto Federal de Educação, Ciência e Tecnologia do Rio Grande do Norte (IFRN), Caicó - Rio Grande do Norte, CEP: 59300-000; E-mail: jcarlosmequi@ hotmail.com

${ }^{5}$ Mestre em Ensino de Ciências e Educação Matemática; Professor Ensino Básico Técnico e Tecnológico de Química, Instituto Federal de Educação, Ciência e Tecnologia do Rio Grande do Norte (IFRN), Caicó - Rio Grande do Norte, CEP: 59300-000; E-mail: carlos.chaves@ifrn.edu.br

${ }^{6}$ Administrador com Habilitação em Marketing, Mestrando em Engenharia Têxtil, Universidade Federal do Rio Grande do Norte (UFRN), Programa de Pós Graduação em Engenharia Têxtil (PPGET), Natal - Rio Grande do Norte, CEP: 59078-970; E-mail: adair.badaro@ ifrn.edu.br ${ }^{7}$ Doutora em Engenharia Agrícola; Técnica de Alimentos, Instituto Federal de Alagoas (IFAL), Piranhas - Alagoas, CEP:57460-000; E-mail: regilanemarques@yahoo.com.br
} 


\section{Introdução}

A polpa de frutas é amplamente usada em vários segmentos da indústria de produtos alimentícios, tais como as indústrias de sucos naturais, sorvetes, laticínios e etc. (Silva et al., 2010). O seu processamento para obtenção de polpas é uma atividade agroindustrial importante, pois agrega valor econômico à fruta e evita desperdícios, minimizando as perdas que podem ocorrer durante a comercialização do produto in natura (Nascimento et al., 2013).

No processamento das polpas de frutas, ocorre a aplicação de processos térmicos que envolvem transferência de calor, como aquecimento, resfriamento e congelamento. Assim, o conhecimento das propriedades termofísicas, como massa específica, se torna, fundamental para o dimensionamento dos equipamentos utilizados nos processos que envolvam transferência de calor, a fim de estimar o tempo de processamento e a quantidade de energia envolvida durante os processos térmicos (Pereira, 2013).

Como a composição dos materiais biológicos é heterogênea, a modelagem e optimização dos processos dependem de parâmetros que podem variar durante o processo como, o teor de umidade, estrutura do produto e temperatura utilizada (Mattos e Medeiros, 2008).

$\mathrm{O}$ efeito da temperatura e concentrações em sólidos solúveis ( ${ }^{\circ}$ Brix) na massa específica em sucos e polpas de frutas tem sido alvo de estudados, como: da polpa de açaí, avaliada, em três concentrações $(9,7 \%, 12 \%$ e $15,2 \%)$ de sólidos solúveis e temperaturas de 10, 20,30, 40 a $50{ }^{\circ} \mathrm{C}$ (Pereira et al., 2002); a polpa de melancia em diferentes temperaturas $(10,20,30,40,50 \mathrm{e}$ $60{ }^{\circ} \mathrm{C}$ ) e diferentes concentrações de sólidos solúveis totais $\left(8,17,26\right.$ e $35^{\circ}$ Brix) (Guedes et al., 2010); do suco de caju em função da temperatura de $5,20,35,50,65$ e $80^{\circ} \mathrm{C}$ (Bonomo et al., 2009), entre outros.

Os valores experimentais da variação da massa específica com a temperatura e concentração da polpa de fruta são muito importantes para as indústrias de alimentos em geral, uma vez que esses dados são essenciais na concepção e optimização de várias operações, como no projeto de bombas, trocadores de calor, evaporadores e misturadores (Ramos e Ibarz, 1998; Pereira et al., 2002).
Os modelos matemáticos para predição das propriedades termofísicas evoluíram e representam uma oportunidade significativa de melhorar a eficiência de tratamentos térmicos no processamento de alimentos, além, de certa forma, se tornar uma alternativa viável na substituição da determinação experimental destes parâmetros, o qual pode ser muito dispendioso (Egea et al., 2015).

Dessa forma, o objetivo desse trabalho foi determinar experimentalmente a massa específica das polpas de abacaxi e acerola e estabelecer uma correlação entre essa propriedade com a variação da temperatura de 10 a $50^{\circ} \mathrm{C}$, assim como a energia de ativação.

\section{Material e Métodos}

As matérias-primas abacaxi (Ananas comosus (L.) Merr) e acerola (Malpighia punicifolia L.) foram oriundas do comércio local da cidade de Caicó do Rio Grande do Norte (IFRN). Os frutos foram transportados e processados no Laboratório de Química do IFRN. Após o recebimento e seleção, os frutos foram lavados em água corrente e imersos em uma solução de hipoclorito de sódio a $50 \mathrm{ppm}$, durante 15 minutos para sanitização. Em seguida, foram enxaguados e despolpados utilizando um multiprocessador, para o devido envasamento em sacos plásticos de baixa densidade e armazenamento em um refrigerador, até o momento das análises.

Para as análises da massa específica das polpas foram utilizados picnômetros previamente calibrados com água destilada, nas temperaturas de $10,20,3040$ e $50^{\circ} \mathrm{C}$, e pesados em balança analítica com precisão de $0,0001 \mathrm{~g}$, As temperaturas das amostras foram controladas através do banho termostato e por meio dos termômetros presentes nos próprios picnômetros. O cálculo da massa específica foi realizado através da Equação 1, que estabelece relação entre massa da polpa e volume do picnômetro.

$\rho=m / v$

Equação 1

onde: $\rho$ - Massa específica do produto $\left(\mathrm{kg} / \mathrm{m}^{3}\right) ; \mathrm{v}-$ Volume do picnômetro $\left(\mathrm{m}^{3}\right) ; \mathrm{m}$ - Massa do produto $(\mathrm{kg})$

Os sólidos solúveis $\left({ }^{\circ}\right.$ Brix $)$ das polpas foram obtidos através de leitura direta com auxílio de um refratômetro portátil modelo RT-30ATC, com escala de 0 a $32^{\circ}$ Brix, devidamente calibrado e 
ajustado a $20{ }^{\circ} \mathrm{C}$ com água destilada, e os resultados expressos em ${ }^{\circ}$ Brix.

$\mathrm{Na}$ modelagem matemática da massa especifica foram utilizados os modelos polinomiais linear, com três e quatro termos (Tabela 1). Já os modelos de Alvarado e Romero (1989) e Ramos e Ibarz (1998), foram utilizados para verificar os ajustes aos dados experimentais, de acordo com a Tabela 1.

Tabela 1 Equações para o cálculo da massa específica de produtos alimentícios

\begin{tabular}{lc}
\hline \multicolumn{1}{c}{ Modelos Matemáticos } & Equações \\
\hline Alvarado e Romero (sucos e polpas de frutas) & $\rho=1002+4,61 \mathrm{~B}-0,460 \mathrm{~T}+7,001 \times 10^{-3} \mathrm{~T}^{2}-9,175 \times 10^{-5} \mathrm{~T}^{3}$ \\
Ramos e Ibarz (suco de pêssego) & $\rho=1006,56-0,5155 \mathrm{~T}+4,1951 \mathrm{~B}+0,0135 \mathrm{~B}^{2}$ \\
Linear & $\rho=\mathrm{a}+\mathrm{bT}$ \\
Três termos & $\rho=\mathrm{a}+\mathrm{bT}+\mathrm{cT}^{2}$ \\
Quatro termos & $\rho=\mathrm{a}+\mathrm{bT}+\mathrm{cT}^{2}+\mathrm{dT}^{3}$ \\
\hline$\rho$ - Massa especifica $\left(\mathrm{kg} / \mathrm{m}^{3}\right) ; \mathrm{T}-$ Temperatura $\left({ }^{\circ} \mathrm{C}\right) ; \mathrm{B}-$ concentração $\left({ }^{\circ} \mathrm{Brix}\right) ; \mathrm{a}, \mathrm{b}, \mathrm{c}$ e d - constantes.
\end{tabular}

Para a obtenção dos valores da energia de ativação $\left(\mathrm{E}_{\mathrm{a}}\right)$ para a massa específica foi empregado o método proposto por Tsen e King (2002), obtido através da Equação (2):

$\rho=\rho o . \operatorname{Exp}\left(E_{a} / R T\right)$

Equação 2

Onde: $\rho_{0}$ é massa específica $\left(\mathrm{kg} / \mathrm{m}^{3}\right) ; \mathrm{E}_{\mathrm{a}}$ : energia de ativação (J/g-mol); R: constante universal dos gases $(8,3144 \mathrm{~J} / \mathrm{g}-\mathrm{mol} \mathrm{K})$; T: temperatura absoluta $(\mathrm{K})$.

Os ajustes dos modelos foram realizados pelo método Quasi-Newton por meio de análises de regressão não linear. O grau de ajuste de cada modelo foi considerado pela magnitude do coeficiente de determinação $\left(\mathrm{R}^{2}\right)$.

Foram feitas regressões lineares dos dados da massa específica para obtenção das equações que representa essa grandeza, utilizando o programa STATISTICA.

\section{Resultados e Discussão}

$\mathrm{Na}$ Tabela 2, têm-se os valores experimentais da massa específica das polpas de abacaxi e acerola nas temperaturas de 10 a $50^{\circ} \mathrm{C}$ e, seus respectivos desvios padrão e coeficiente de variação. Os valores das massas específicas das polpas de abacaxi e acerola variaram de 1060,31 a $1044,32 \mathrm{~kg} / \mathrm{m}^{3}$ e 1064,55 a $1053,91 \mathrm{~kg} / \mathrm{m}^{3}$, respectivamente. Estes valores estão próximos aos valores de massa específica obtida para o suco de laranja, nas concentrações de 10 e $20^{\circ}$ Brix, iguais a 1057,24 a $1046,04 \mathrm{~kg} . \mathrm{m}^{-3}$ e 1086,01 a $1073,57 \quad \mathrm{~kg} \cdot \mathrm{m}^{-3}$, respectivamente, nas temperaturas de 10 a $50^{\circ} \mathrm{C}$ (Ramos e Ibarz, 1998).

Tabela 2 Valores médios da massa específica da polpa de Abacaxi nas temperaturas de 10 a $50{ }^{\circ} \mathrm{C}$, desvio padrão e coeficiente de variação

\begin{tabular}{ccccc}
\hline Polpas de frutas & Temperatura $\left({ }^{\mathbf{C}} \mathbf{C}\right)$ & Massa específica $\left(\mathbf{k g} / \mathbf{m}^{\mathbf{3}}\right)$ & Desvio padrão & Coeficiente de variação $(\%)$ \\
\hline & 10 & 1060,31 & 0,544 & 0,051 \\
Abacaxi & 20 & 1058,43 & 0,428 & 0,040 \\
$14,4^{\circ}$ Brix & 30 & 1054,01 & 0,255 & 0,024 \\
& 40 & 1047,61 & 0,486 & 0,046 \\
& 50 & 1044,32 & 0,500 & 0,048 \\
\hline & 10 & 1064,55 & 0,305 & 0,029 \\
Acerola & 20 & 1063,34 & 0,486 & 0,046 \\
$17,0^{\circ}$ Brix & 30 & 1061,22 & 0,246 & 0,023 \\
& 40 & 1059,61 & 0,268 & 0,025 \\
\hline
\end{tabular}

Observa-se também que os valores médios das massas específicas obtidas para a polpa de abacaxi e polpa de acerola diminuíram com o aumento da temperatura. Bonomo et al., (2009) estudando o comportamento da massa específica do suco de caju em função da temperatura ( 5 a 80
${ }^{\circ} \mathrm{C}$ ), observaram diminuição da massa específica com o aumento da temperatura. A redução da massa específica das polpas estudadas com o aumento da temperatura deve ter sido devido à expansão térmica que ocorre com as polpas de frutas quando são aquecidas. Diniz et al., (2014) 
verificaram que os valores de massa específica da polpa de goiaba diminuíram com o aumento da temperatura devido à expansão volumétrica do fluido.

Comparando os valores de massa específica da polpa de abacaxi com a polpa de acerola, nota-se que os valores da massa específica da polpa de abacaxi são menores nas mesmas temperaturas aplicadas. Provavelmente, devido à polpa de abacaxi ter menores valores de sólidos solúveis $\left(14,5^{\circ}\right.$ Brix) em relação à polpa de acerola $(17,0$ ${ }^{\circ}$ Brix). Segundo Guedes et al. (2010), estudando o comportamento da massa específica da polpa de melancia em diferentes temperaturas $(10,20,30$, 40,50 e $\left.60{ }^{\circ} \mathrm{C}\right)$ e concentrações $(8,17,26$ e 35
${ }^{\circ}$ Brix), observaram que a massa específica aumenta à medida que ocorre a concentração de sólidos solúveis e diminui com elevação da temperatura. O mesmo comportamento foi observado por Giraldo et al. (2017) para a massa específica do suco clarificado de groselha, entre as concentrações de 20 a $50^{\circ}$ Brix e temperaturas de 10 a $50^{\circ} \mathrm{C}$.

$\mathrm{Na}$ Tabela 3, encontram-se as equações polinomiais que foram utilizadas para descrever $o$ efeito da temperatura sobre a variação da densidade das polpas de abacaxi e acerola e, seus respectivos coeficientes de determinação obtidos por meio de análise de regressão.

Tabela 3 Equações propostas para o cálculo da massa específica das polpas de abacaxi e acerola em função da temperatura

\begin{tabular}{ccccccc}
\hline Polpa de fruta & Equações polinomiais & $\mathbf{a}$ & $\mathbf{b}$ & $\mathbf{c}$ & $\mathbf{d}$ & $\mathbf{R}^{\mathbf{2}}$ \\
\hline \multirow{3}{*}{ Abacaxi } & $\rho=\mathrm{a}+\mathrm{bT}$ & 1065,776 & $-0,42800$ & - & - & 0,9863 \\
& $\rho=\mathrm{a}+\mathrm{bT}+\mathrm{cT}^{2}$ & 1063,376 & $-0,22229$ & $-0,00343$ & - & 0,9907 \\
& $\rho=\mathrm{a}+\mathrm{bT}+\mathrm{cT}^{2}+\mathrm{dT}^{3}$ & 1055,37 & 0,8889 & $-0,0458$ & $0,471 \times 10^{-3}$ & 0,9984 \\
\hline \multirow{3}{*}{ Acerola } & $\rho=\mathrm{a}+\mathrm{bT}$ & 1068,029 & $-0,2501$ & - & - & 0,9507 \\
& $\rho=\mathrm{a}+\mathrm{bT}+\mathrm{cT}^{2}$ & 1063,794 & 0,1129 & $-0,00605$ & - & 0,9889 \\
& $\rho=\mathrm{a}+\mathrm{bT}+\mathrm{cT}^{2}+\mathrm{dT}^{3}$ & 1068,25 & $-0,515$ & 0,0178 & $-0,27 \times 10^{-3}$ & 0,9925 \\
\hline
\end{tabular}

$\rho$ - Massa especifica $\left(\mathrm{kg} / \mathrm{m}^{3}\right) ; \mathrm{T}$ - Temperatura $\left(10\right.$ a $\left.50^{\circ} \mathrm{C}\right)$; a, b, c e d - constantes, $\mathrm{R}^{2}$ coeficiente de determinação

Observa-se que as três equações podem ser utilizadas para predizer a massa específicas das duas polpas de frutas, embora os modelos polinomiais com três termos possuam os maiores coeficientes de determinação $\left(R^{2}>0,99\right)$, sendo aquele que melhor se ajustaram aos dados experimentais. Lima et al. (2002) estudando o ajuste da massa específica da polpa de cupuaçu com as equações do tipo linear, quadrática e polinomial de quatro termos concluíram que a equação polinomial de quatro termos apresentou melhor ajuste, pois apresentaram coeficientes de determinação $\left(R^{2}\right)$ superiores a 0,99 .

Lima et al. (2003), propuseram equações polinomiais do tipo linear e quadrática para calcular a massa específica da polpa de umbu com 10,20 e $30^{\circ}$ Brix, em função da temperatura (20, 30 e $40^{\circ} \mathrm{C}$ ). Pereira et al. (2002), avaliaram a massa específica de polpa de açaí em três concentrações de sólidos totais $(9,7 \%, 12 \%$ e $15,2 \%)$ e diferentes temperaturas $\left(10^{\circ} \mathrm{C}\right.$ a $\left.50^{\circ} \mathrm{C}\right)$, constataram um decréscimo da massa específica com o aumento da temperatura da polpa em todas as concentrações. Os mesmos observaram que a equação quadrática resultou nos melhores coeficientes de determinação $\left(\mathrm{R}^{2}\right)$ em todas as amostras. Minim et al. (2009), analisando o comportamento da massa específica do suco do limão em relação a temperatura e sua concentração, perceberam que o modelo polinomial se ajustou satisfatoriamente aos resultados experimentais, cujo coeficiente de determinação foi de 0,99 .

Nas Figuras 1 e 2, tem-se as curvas experimentais e teóricas das massas específicas obtidas para as polpas de abacaxi e acerola, respectivamente, em função da temperatura. Os valores teóricos foram calculados conforme os modelos Alvarado e Romero (1989), proposto para polpas de frutas em geral, e Ramos e Ibarz (1998), proposto para suco de pêssego, e através das três equações propostas anteriormente.

Nota-se que as equações utilizadas para os cálculos das massas específicas das polpas de abacaxi e acerola, em função da temperatura, ajustaram-se melhor aos dados experimentais que os modelos propostos por Ramos e Ibarz (1998) e Alvarado e Romero (1989). 


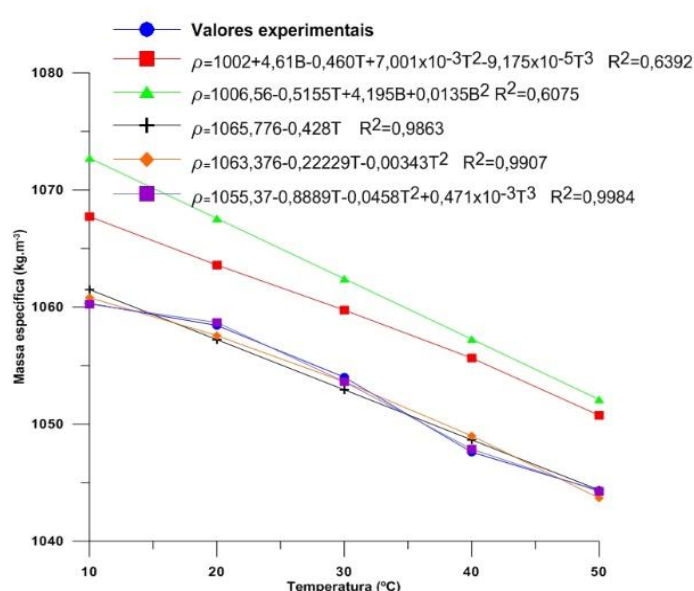

Figura 1 Valores experimentais e teóricos da massa específica da polpa de abacaxi em função da temperatura.

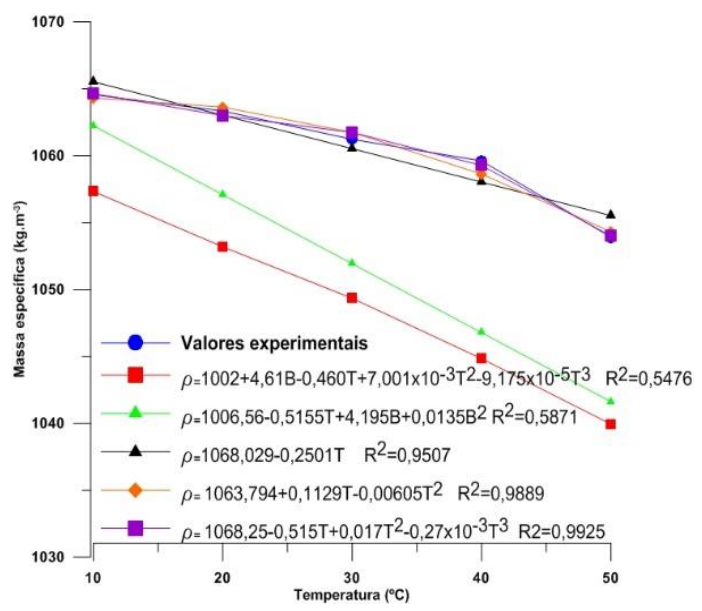

Figura 2 Valores experimentais e teóricos da massa específica da polpa de acerola em função da temperatura.

Verificam-se na Tabela 4, os valores dos parâmetros do modelo de Arrhenius calculado a partir da relação da massa específica com a variação da temperatura para as polpas de abacaxi e acerola.

Tabela 4 Valores dos parâmetros do modelo de Arrhenius calculado a partir da relação da massa específica

\begin{tabular}{cccc}
\hline $\begin{array}{c}\text { Polpa de } \\
\text { frutas }\end{array}$ & $\begin{array}{c}\boldsymbol{\rho o}_{0} \\
\left(\mathbf{k g} / \mathbf{m}^{\mathbf{3}}\right)\end{array}$ & $\begin{array}{c}\text { Ea } \\
(\mathbf{J} / \mathbf{g m o l})\end{array}$ & $\mathbf{R}^{\mathbf{2}}$ \\
\hline Abacaxi & 920,6895 & 338,11 & 0,9861 \\
Acerola & 981,0468 & 196,25 & 0,9503 \\
\hline
\end{tabular}

$\rho_{0}$ - massa específica; Ea:- energia de ativação; $R^{2}$ coeficiente de determinação

Observa-se que o valor da massa específica inicial da acerola foi maior que a da polpa do abacaxi, enquanto ocorreu o inverso para energia de ativação. $\mathrm{O}$ valor superior da energia de ativação para a massa específica da polpa de abacaxi indica maior sensibilidade da polpa de abacaxi ao aplicar diferentes temperaturas, em comparação a polpa de acerola. Esse fato também pode estar relacionado às características individuais de cada fruto.

Os valores encontrados para a energia de ativação das polpas de abacaxi e acerola são inferiores a energia de ativação do purê de banana, encontrado por Tsen e King (2002), obtiveram valores variando de 1137,36 a 748,36 J/g-mol, com as concentrações de sólidos solúveis variando de 10 a $80^{\circ}$ Brix e temperaturas de 5 a $80{ }^{\circ} \mathrm{C}$.

\section{Conclusão}

Os valores das massas específicas diminuíram com o aumento da temperatura; o modelo polinomial com três termos apresentou os maiores coeficientes de determinação $\left(R^{2}>0,99\right)$, podendo ser usado para estimar a massa específica das polpas estudadas.

A polpa de abacaxi apresentou maior sensibilidade à aplicação das diferentes temperaturas.

\section{Referências}

Alvarado, J. D.; Romero, C. H. Physical properties of fruits-I-II: density and viscosity of juices as functions of soluble solids and content and temperature. Latin American Applied Research, v.19, p.15-21, 1989.

Araújo, J. L.; Queiroz, A. J. M.; Figueirêdo, R. M. F. Massa específica de polpa de cupuaçu (theobroma grandiflorum schum.) sob diferentes temperaturas. Revista Brasileira de Produtos Agroindustriais, v.4, n.2, p.127-134, 2002. 8595/rbpa.v4n2p127-134 http://dx.doi.org/10.15871/1517-

Bonomo, R. C. F.; Fontan, R. C. I.; Souza, T. S.; Veloso, C. M.; Reis, M. F. T.; Castro, S. S. Thermophysical properties of cashew juice at different concentrations and temperatures. Revista Brasileira de Produtos Agroindustriais, v.11, n.1, p.35-42, 2009. http://www.deag.ufcg.edu.br/rbpa/rev111/Art1116.pdf

Dantas Júnior, E. E.; Queiroz, A. J. M.; Figueiredo, R. M. F. Determinação e elaboração de modelos de predição da massa específica da 
manga espada. Revista Educação Agrícola Superior, v.22, n.1, p.39-42, 2007.

Diniz, R. S.; Coimbra, J. S. R.; Martins, M. A.; Santos, M. O.; Diniz, M. D. M. S.; Santos, E. S.; Santánna, D. D.; Rocha, R. A.; Oliveira, E. B. Physical Properties of Red Guava (Psidium guajava L.) Pulp as Affected by Soluble Solids Content and Temperature. International Journal of Food Engineering, v.10, n.3, p.437445, 2014. https://doi.10.1515/ijfe-2012-0250

Egea, M. B.; Reis, M. H. M.; Danesi, E. D. G. Aplicação de modelos matemáticos preditivos para o cálculo das propriedades termofísicas do palmito pupunha. Revista Brasileira de Produtos Agroindustriais, v.17, n.2, p.179187, 2015. http://www.deag.ufcg.edu.br/rbpa/rev172/Art1728.pdf

Giraldo, G. I; Cruz, C. D.; Sanabria, N. R. Propriedades físicas del jugo de uchuva (Physalis peruviana) clarificado en Función de la Concentración y la Temperatura. Información tecnológica, v.28, n.1, p.133-142, 2017. http://dx.doi.org/10.4067/S071807642017000100013.

Guedes, D. B.; Ramos, A. M.; Diniz, M. D. M. S. Efeito da temperatura e da concentração nas propriedades físicas da polpa de melancia. Brazilian Journal Food Technology, v.13, n.4, p.279-285, 2010. http://bjft.ital.sp.gov.br/artigos/html/busca/PDF /v13n4433a.pdf

Lima, Í. J. E.; Queiroz, A. J. M.; R. M. F. Figueirêdo. Propriedades termofísicas da polpa de umbu. Revista Brasileira de Produtos Agroindustriais, Especial, n.1, p.31-42, 2003. http://www.deag.ufcg.edu.br/rbpa/rev5e/Art5e4.pdf

Mattos, J. S.; Mederos, B. J. T. Densidade de polpas de frutas tropicais: banco de dados e determinação experimental. BioEngenharia, v.2, n.2, p.109-118, 2008. http://dx.doi.org/10.18011/bioeng2008v2n2p109-118

Minim, L. A.; Telis, V. R. N.; Minim, V. P. R.; Alcantara, L. A. P.; J. Telis-Romero. Thermophysical properties of lemon juice as affected by temperature and water content. Journal of Chemical \& Engineering Data, v.54, n.8, p.2269-2272, 2009. https://pubs.acs.org/doi/abs/10.1021/je900155c

Nascimento, J. M. C.; Moura, M. F. V.; Fonseca, P. A. Q.; Cruz, A. M. F.; Soares, J. C. Determinação do Teor de Ácido Ascórbico pelo Método de Tillmans em Polpas de Frutas. In: LIII Congresso Brasileiro de Química, 53, Rio de Janeiro, 2013. Anais. Rio de Janeiro: Associação Brasileira de Química. http://www.abq.org.br/cbq/2013/trabalhos/4/308016647.html

Pereira, E. A.; Queiroz, A. J. M.; Figueiredo, R. M. F. Massa específica de polpa de açaí em função do teor de sólidos totais e da temperatura. Revista Brasileira de Engenharia Agrícola e Ambiental, v.6, n.3, p.526-530, 2002. http://dx.doi.org/10.1590/S1415$\underline{43662002000300025}$

Pereira, C. G. Propriedades termofísicas e comportamento reológico de polpa de acerola em diferentes concentrações e temperaturas. 2013, 135 f. Dissertação (mestrado em Ciência dos Alimentos), Universidade Federal de Lavras, Lavras, 2013.

Ramos, A. M.; Ibarz, A. Density of juice and fruit puree as a function of soluble solids content and temperature. Journal of Food Engineering, v.35, n.1, p.57-63, 1998. https://doi.org/10.1016/S0260-8774(98)00004-1

Silva, M. A. C.; Silva, Z. E.; Oliveira, E. P.; Oliveira, A. S. S.; Silva, C. K. F. Determinação de propriedades termofísicas da polpa de acerola: aplicação do método flash. In: VI Congresso Nacional de Engenharia mecânica, 4, Campina Grande, 2010. Anais. Campina Grande: Universidad Federal de Campina Grande, p.1-7. http://www.abcm.org.br/anais/conem/2010/PDF/CON101544.pdf.

Tsen, J. H.; King, V. A. E. Density of banana puree as a function of soluble solids concentration and temperature. Journal of Food Engineering, v.55, n.4, p.305-308, 2002. https://doi.org/10.1016/S0260-8774(02)00105-X 